\title{
Environmental effects and repeatability estimates for sperm production and semen quality of Holstein bulls
}

\author{
Ismaïl Boujenane and Khouloud Boussaq \\ Department of Animal Production and Biotechnology, Institut Agronomique et Vétérinaire Hassan II, Rabat, Morocco
}

\begin{abstract}
The purpose of this study was to estimate environmental effects on semen production of artificial insemination (Al) Holstein bulls managed under Moroccan conditions. A total of 4046 ejaculates collected from 34 Holstein bulls in the years 2009-2013 were analysed. Studied variables were volume, concentration, total number of spermatozoa, mass motility, individual motility and post-thawing motility. Data were analysed by REML method using the mixed model including the random effect of bull and the fixed effects of age at collection, season of collection, year of collection, interval between two collections and ejaculate order. The effect of age of bulls was significant for all studied variables, except for mass motility. Spring and winter were the best seasons for sperm production and quality. Bulls' ejaculates collected once a day at one day interval produced monthly $30 \%, 86 \%, 156 \%, 183 \%$ and $185 \%$ more motile spermatozoa than those collected once a day at 2, 3, 4, 5 and 6 days interval, respectively. Moreover, bulls' sperm collected twice a day at 1, 2, 3, 4, 5 and 6 days interval produced monthly (sum of motile spermatozoa obtained at 1st and 2nd ejaculates) $77 \%$, $70 \%, 65 \%, 68 \%, 84 \%$ and $91 \%$ more than those collected once a day at the same interval, respectively. Repeatability estimates for semen traits were medium to high. They varied from 0.157 for mass motility to 0.411 for ejaculate volume. It was concluded that environmental factors clearly contribute to semen production in Holstein bulls and short intervals between collections and two collections per day are maximising sperm production.
\end{abstract}

Keywords: ejaculate, semen, spermatozoa, artificial insemination, volume, motility, repeatability 


\section{Introduction}

Moroccan dairy herds are experiencing a rapid expansion of artificial insemination ( $\mathrm{Al})$. Although less than $10 \%$ of the national cow herds are artificially inseminated, the number of Al has increased during the last two decades by about $31 \%$ per year. In Morocco, Al concerns mainly the improved dairy cows, with Holstein Al representing more than $68 \%$ (Boujenane 2002). Also, there are two Al centres in the country that are managed by the government. Their production does not cover the increased Al demand; therefore thousands of frozen doses are imported annually from European and North-American countries. Recently, one Al centre was transferred to a breeders' association, which hopes to increase the local production in order to satisfy the breeders' Al demand. Moreover, most of the bulls used in the centre have been imported from Europe, i.e. developed in temperate areas. Thus, there is a need for extensive knowledge of factors affecting sperm production and semen quality obtained under Moroccan management conditions in order to increase the productivity and profitability of the Al centre. Environmental factors that affect semen production are mainly age of the bull at semen collection, frequency of collection and season of collection, the latter e.g. including food quality, ambient temperature, humidity or day length (Everett \& Bean 1982, Taylor et al. 1985, Mathevon et al. 1998, Karoui et al. 2011). Indeed, with the knowledge of these factors, the centre might adapt management of Al bulls to improve semen production.

The purpose of this study was to estimate effects of age, season, year and frequency of collection on semen characteristics and repeatability for semen traits of Al Holstein bulls managed and housed under Moroccan conditions.

\section{Material and methods}

\section{Animals and management}

Semen data from a Moroccan AI centre, CRIA Fouarat, collected in the years 2009-2013 were evaluated. Average daily ambient temperature during the collection period was $16.3^{\circ} \mathrm{C}$ with a minimum of $-3.7^{\circ} \mathrm{C}$ and a maximum of $42.8^{\circ} \mathrm{C}$. Average humidity was $77 \%$. A total of 4046 ejaculates were analysed. They were obtained from 34 Holstein bulls of which 30 were imported from France and four were born in Morocco. Age of bulls at collection averaged 39.5 month, varying from 13 to 93 months. The bulls were managed uniformly. They were individually housed and maintained on a diet of mixed alfalfa and oat hay, approximately $500 \mathrm{~g}$ of concentrate and vitamins and minerals mix. All the bulls are periodically vaccinated against foot and mouth disease.

\section{Semen collection}

In general, semen was routinely collected in the centre three times a week (Monday, Wednesday and Friday) at early morning. Semen collection was preceded by a controlled sexual preparation. Thus, after arrival on the collection floor, bulls were tied in the waiting stall from which they observed the collection of the other bulls. When their libido was stimulated, they were allowed a short walk and two false mounts with a 2-min interval. At the third mount using a teaser animal (a bull known for its docility and robustness), the ejaculate 
was obtained with an artificial vagina $\left(37^{\circ} \mathrm{C}\right)$. One or two ejaculates were collected from each bull on each collection day. Ejaculates obtained from the 1st and 2nd collection represented $60.8 \%$ and $39.2 \%$, respectively. The interval between two consecutive collections varied from 1 to 36 days, with an average of 3.33 days.

\section{Semen exam}

Immediately after collection, the tube of semen was placed in a water bath at 32 to $34^{\circ} \mathrm{C}$. Each ejaculate was evaluated for volume, sperm concentration and motility traits. Some ejaculates were discarded by the technician on either established standards or judgment (colour, presence of blood or dirt...). Volume was measured in the graduated collection tube $(\mathrm{ml})$. Sperm concentration (spermatozoa number per $\mathrm{ml}$ ) was estimated by using a photometer (Reference 1203, IMV Technologie, France). Mass motility score was subjectively assessed for undiluted unstained semen using a scale from 0 (no activity) to 5 (rapid swirling motion). Individual ejaculates were then diluted with egg yolk citrate-glycerol extender to give a concentration of 24 million total spermatozoa per $0.25 \mathrm{ml}$ straw. Individual motility or percent progressive motility, expressed by the percent of mobile spermatozoa per ejaculate, was estimated in $5 \%$ steps on a subjective scale of $0 \%$ to $100 \%$ by examining unstained diluted semen under microscope (Nikon Eclipse 50i, IMV Technologie, France) using a 100X magnification. After gradually cooling to $4^{\circ} \mathrm{C}$, the semen was packaged in $0.25 \mathrm{ml}$ straws (24 million motile spermatozoa before freezing per straw) and frozen at $-196^{\circ} \mathrm{C}$. Postthawing motility was measured 24 hours after storage in liquid nitrogen. For post-thaw semen evaluation, two straws from each bull at each collection were thawed in a water bath of $37^{\circ} \mathrm{C}$ for 30 seconds and evaluated individually for the percentage of progressively motile spermatozoa. Ejaculates with less than $35 \%$ of post-thawing progressively motile spermatozoa were eliminated. Valid straws were stored in large containers containing liquid nitrogen.

\section{Statistical analyses}

The traits studied were ejaculate volume, sperm concentration, mass motility score, individual motility, post-thawing progressive individual motility and total number of spermatozoa per ejaculate, which was calculated as the product of ejaculate volume and sperm concentration. After editing, the file analysed included 4046 ejaculates corresponding to 34 bulls. The number of ejaculates of each bull averaged 119, varying from 7 to 349 ejaculates. Except mass motility score that was analysed using the GLIMMIX (SAS Institute Inc., Cary, NC, USA) procedure (SAS 2002), since it is multinomially distributed, the other semen traits (ejaculate volume, sperm concentration, total number of spermatozoa per ejaculate, individual motility and post-thawing individual motility), which are normally distributed, were analysed using the MIXED procedure (SAS 2002). The mixed model used to analyse all variables included the random effect of bull ( 34 bulls) and the fixed effects of age at collection ( 7 levels: age $\leq 24 \mathrm{mo}$., $24<$ age $\leq 30$ mo., $30<$ age $\leq 36$ mo., $36<$ age $\leq 42$ mo., $42<$ age $\leq 48$ mo., $48<$ age $\leq 54$ mo., age $>54$ mo.), season of collection (4 levels: winter: January - March, spring: April - June, summer: July - September, autumn: October - December), year of collection (5 levels: 2009, 2010,..., 2013), interval between two successive collections ( 6 levels: $1,2, \ldots, 6$ or greater) and ejaculate order 
(2 levels: 1st and 2nd). Interactions between effects were assumed negligible and hence were not tested. When the effect was determined to be significant $(P<0.05)$, differences among least-squares means were examined by the Tukey method for multiple comparisons.

Repeatability estimates for semen traits were calculated by using the following formula:

$$
\frac{\sigma^{2} b u l l}{\sigma^{2} b u l l+\sigma^{2} e r r o r}
$$

The variance components $\sigma^{2} b u l l$ and $\sigma^{2}$ error were estimated from the MIXED procedure (SAS 2002).

\section{Results and discussion}

\section{Descriptive statistics}

Arithmetic means, standard deviations and coefficients of variation of sperm characteristics of Holstein bulls' semen collected in Moroccan conditions are reported in Table 1. They are in general similar to those found in other studies (Everett \& Bean 1982, Taylor et al. 1985, Mathevon et al. 1998, Karoui et al. 2011). Moreover, ejaculate volume had the highest and individual motility had the lowest coefficient of variation.

Table 1

Number of observations, arithmetic means, standard deviations and coefficients of variation of sperm production and semen quality of Holstein bulls

\begin{tabular}{lcccc}
\hline Semen trait & Number & $\begin{array}{c}\text { Arithmetic } \\
\text { mean }\end{array}$ & $\begin{array}{c}\text { Standard } \\
\text { deviation }\end{array}$ & $\begin{array}{c}\text { Coefficient } \\
\text { of variation, \% }\end{array}$ \\
\hline Ejaculate volume, $\mathrm{ml}$ & 4046 & 5.05 & 2.25 & 44.5 \\
Sperm concentration, $\times 10 \% / \mathrm{ml}$ & 4046 & 0.95 & 0.21 & 22.1 \\
Number of spermatozoa per ejaculate, $\times 10^{9}$ & 4046 & 4.90 & 2.63 & 53.7 \\
Mass motility score $(1-5$ scale) & 4046 & 3.58 & 0.50 & 14.0 \\
Individual motility, \% & 4046 & 77.0 & 5.58 & 7.25 \\
Post-thawing motility, \% & 4046 & 59.8 & 5.82 & 9.73 \\
\hline
\end{tabular}

\section{Systematic environmental effects}

\section{Age at collection}

Age of bulls affects all semen traits $(P<0.01$ to $P<0.001)$ except mass motility score $(P>0.05)$ (Table 2). Ejaculate volume and total number of spermatozoa increased from the youngest to the oldest ages by $54.7 \%$ and $33.5 \%$, respectively, whereas the largest difference for sperm concentration $(10 \times 109 \times \mathrm{xml}-1)$ was recorded between $24<$ age $\leq 30$ and $48<$ age $\leq 54$ classes. Also, individual motility and post-thawing motility differed significantly with age of bulls, but they showed a different pattern with a tendency to a slow decline as the age increased for ages greater than 54 months. These results are in agreement with those of most studies, which reported an increase in semen production and quality with age of bull (Everett \& Bean 1982, Taylor et al. 1985, Mathevon et al. 1998, Brito et al. 2002, Fuerst-Waltl et al. 2006). Moreover, Mathevon et al. (1998) found that volume and number of spermatozoa per ejaculate tended to increase with age of the bull, regardless of season or interval between collections. It seems that the main factor, determining the total number of spermatozoa produced, is the increased ejaculate volume owing to testicular development, since the size 

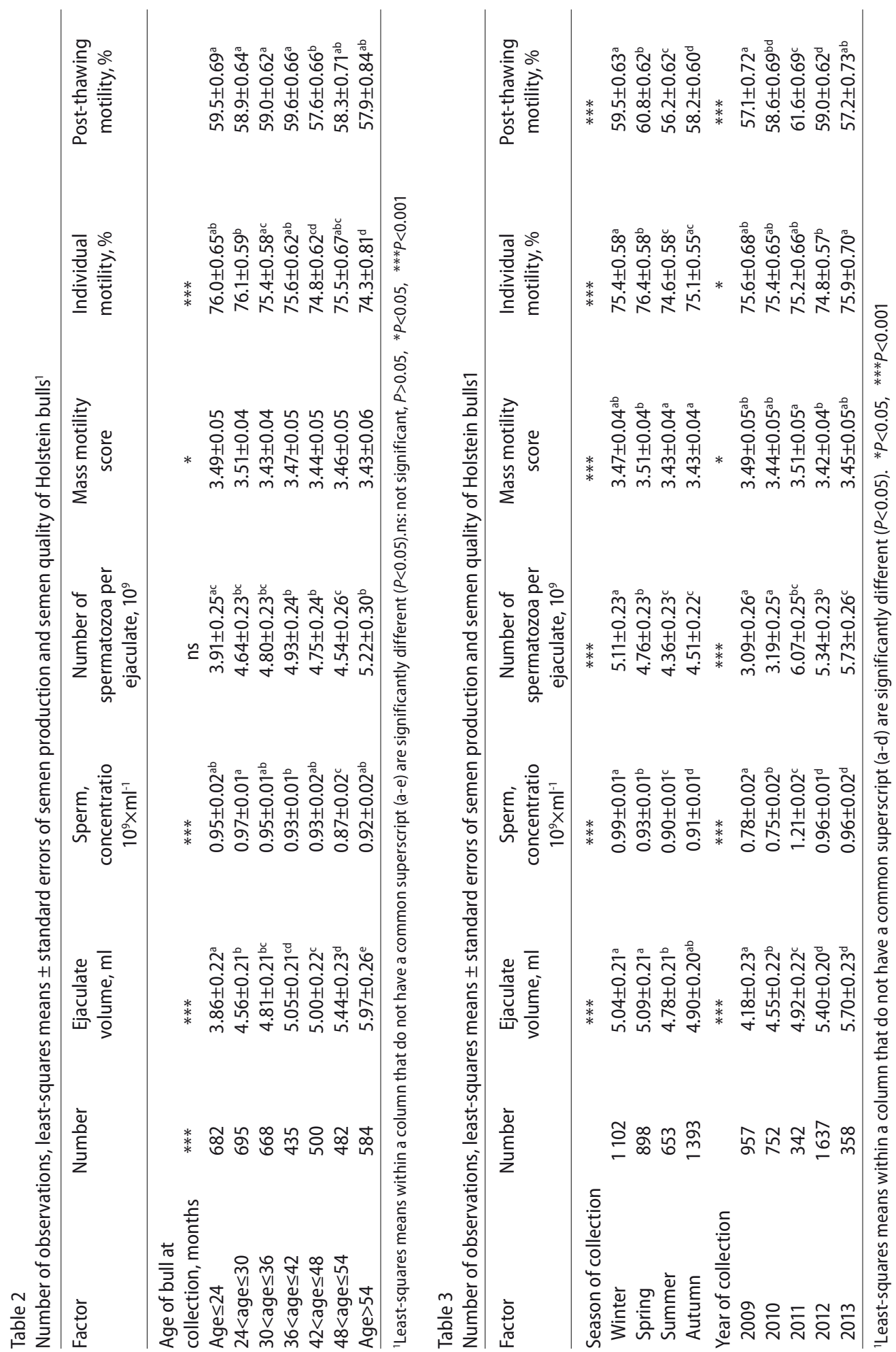
of testes increases for at least five years after puberty (Amann \& Almquist 1976). However, our results are not in agreement with those of Brito et al. (2002), who reported a non-significant effect of age on sperm concentration and motility.

\section{Season of collection}

The season at which semen was collected affects all semen traits of bulls $(P<0.001)$. Semen characteristics were in general higher during spring and winter than during summer and autumn. Ejaculate volume, sperm concentration, total number of spermatozoa, mass motility score, individual motility and post-thawing motility of bulls' sperm collected in winter were $5.44 \%, 10.0 \%, 17.2 \%, 1.17 \%, 1.07 \%$ and $5.87 \%$, respectively higher than those collected in summer. Many studies have reported on seasonal effects on semen production (Everett \& Bean 1982, Mathevon et al. 1998), whereas other investigations failed to detect an effect of season (Brito et al. 2002). The present findings are consistent with those of Mathevon et al. (1998) who found higher values of semen characteristics during winter and spring, but are contrary to those of Taylor et al. (1985) who reported that sperm production in Holstein bulls (ejaculate volume, sperm concentration and total sperm number) was greater during the summer in temperate environments. Moreover, Teixeira et al. (2011) reported that for fresh semen, the ejaculate in April had less volume and sperm concentration, while with the frozen/thawed semen, the proportion of sperm was greater in April to July, decreasing from October to December. Thus, they suggested that reproduction with natural mating should be successful at any time of the year, but the best time of year for using frozen semen is from June to September. Seasonal effects may be due to various factors such as temperature, humidity, photoperiod, feed composition and management. The decrease in sperm production of Holstein bulls housed in Morocco during summer season may be explained by the high temperature recorded during this season. However, Everett \& Bean (1982) and Brito et al. (2002) did not find any significant effects of ambient temperature or humidity on sperm production and semen quality. Likewise, Taylor et al. (1985) demonstrated that extreme temperatures $\left(-24\right.$ to $-19^{\circ} \mathrm{C}$ and 27 to $32^{\circ} \mathrm{C}$ ) had only minor detrimental effects on semen production.

\section{Year of collection}

Semen traits were significantly influenced by the year of collection $(P<0.001)$. Ejaculate volume has increased over years; but no clear trend was observed for the other semen characteristics. These results are in accordance with those of Karoui et al. (2011). The significant differences in semen production between years may be due to changes that are usually observed in e.g. feed quantity and quality, climatic conditions, management practice, equipment, freezing protocol or collectors and technicians.

\section{Interval between collections}

The number of days between two collections affected all studied traits $(P<0.001)$ (Table 4$)$. Large effects of collection interval were also found by Everett \& Bean (1982), Mathevon et al. (1998), Fuerst-Waltl et al. (2006) and Karoui et al. (2011). Increasing collection intervals resulted in significant higher semen characteristics. However, when the length between two collections was four days, ejaculate volume and motility declined slightly, whereas sperm concentration and total number of spermatozoa continued to increase. 


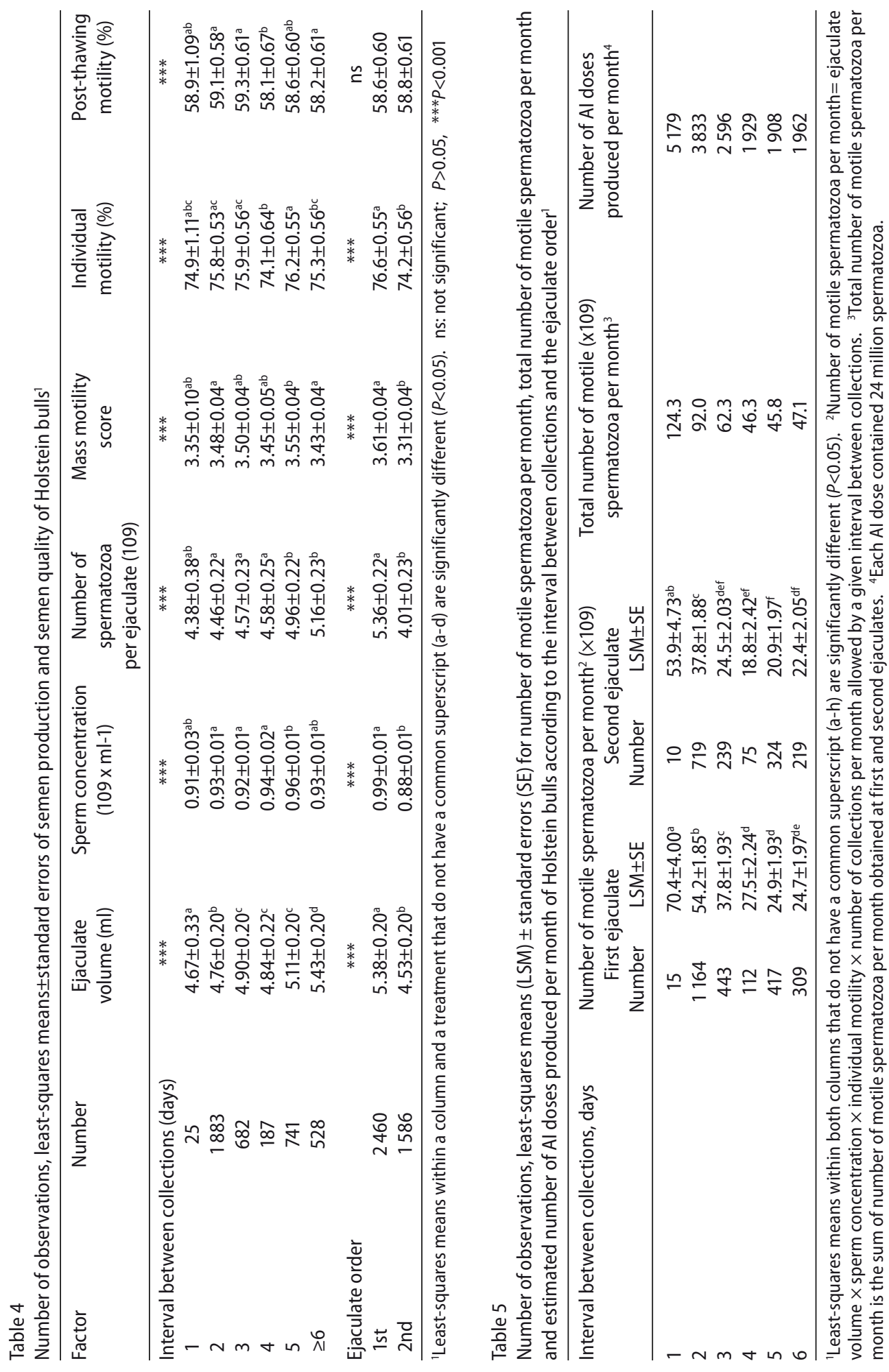




\section{Ejaculate order}

Differences between first and second ejaculates were significant for all studied semen traits $(P<0.01$ to $P<0.001)$, except for post-thawing motility $(P>0.05)$. In general, higher semen quantity and quality was observed for first ejaculates. Differences were $0.85 \mathrm{ml}, 0.11 \times 109 \mathrm{per}$ $\mathrm{ml}, 1.35 \times 109$ spermatozoa, $2.4 \%$ and 0.30 for volume, sperm concentration, total number of spermatozoa per ejaculate, individual motility and mass motility score, respectively. Ejaculate volume, sperm concentration and hence total number of spermatozoa per ejaculate were the characteristics most affected by ejaculate order, since first ejaculates were $18.8 \%, 12.5 \%$ and $33.7 \%$ higher than second ejaculates. Similarly, several researchers (Everett \& Bean 1982, Taylor et al. 1985, Fuerst-Waltl et al. 2006, Karoui et al. 2011) reported that second ejaculates were inferior in sperm quantity and quality, but not with the same importance for all traits. Differences due to collection order may be explained by differences in intensities of sexual preparation and stimulation of bulls prior to each ejaculate.

To answer to the question asked by the centre's manager concerning the optimal interval between two collections for a maximum number of straws, the number of motile spermatozoa produced per month was calculated (ejaculate volume $\times$ sperm concentration $x$ individual motility $\times$ number of collections per month allowed by a given interval between collections). It was assumed that when the interval between two collections was 1, 2, 3, 4, 5 and 6 days; the numbers of collections allowed within five working days per week were $22,13,9,7,5$ and 5 collections during a month period, respectively. Thus, the analysis of number of motile spermatozoa produced per month, by adding the interval between two collections $\times$ ejaculate order interaction to the mixed model used previously, showed that bulls collected once a day at one day interval produced per month $30 \%, 86 \%, 156 \%, 183 \%$ and $185 \%$ more motile spermatozoa than those collected once a day at 2, 3, 4, 5 and 6 days interval, respectively (Table 5). Bulls' sperm collected twice a day (sum of motile spermatozoa obtained at 1st and 2nd collections) at 1, 2, 3, 4, 5 and 6 days interval produced per month $77 \%, 70 \%, 65 \%, 68 \%, 84 \%$ and $91 \%$ more than those collected once a day at the same interval, respectively. The current results support a short interval between collections and two collections per day for maximising sperm production. These findings are in agreement with those of Mathevon et al. (1998).

\section{Repeatability estimates}

Estimates of repeatability for semen traits of Holstein bulls were medium to high. They varied from 0.157 for mass motility score to 0.411 for ejaculate volume (Table 6 ). The higher repeatability estimates indicate that bulls tend to have the same performance for traits with high estimates. Taylor et al. (1985) reported that repeatability estimates for volume, concentration and number of spermatozoa per ejaculate were $0.26,0.23$ and 0.37 , respectively. In addition, Mathevon et al. (1998) found that repeatability estimates varied with age of bulls. They were higher for mature bulls (from 0.51 to 0.64 ) than for young bulls ( 0.41 to 0.53 ) owing to their more stabilized semen production.

In conclusion, this study showed that semen production and sperm quality of Holstein bulls housed in Moroccan conditions were similar to those of bulls raised in temperate conditions. Better adaptation of Holstein bulls to environmental conditions could have been responsible 
for this conclusion. Also, semen characteristics were mostly affected by the environmental factors. Volume and concentration were more affected by age, season and ejaculate order. This study allowed the confirmation that the highest number of Al doses were obtained from short intervals between collections and a high number of collections per day.

Table 6

Variance components and repeatability estimates for semen traits of Holstein bulls

\begin{tabular}{lccc}
\hline Semen traits & $\sigma_{\text {bull }}^{2}$ & $\sigma_{\mathrm{e}}^{2}$ & Repeatability estimates \\
\hline Ejaculate volume & 1.204 & 1.724 & 0.411 \\
Sperm concentration & 0.004 & 0.021 & 0.175 \\
Number of spermatozoa per ejaculate & 1.497 & 2.334 & 0.391 \\
Mass motility score & 0.037 & 0.199 & 0.157 \\
Individual motility & 8.202 & 23.754 & 0.257 \\
Post-thawing motility & 10.368 & 21.197 & 0.328 \\
\hline
\end{tabular}

\section{Acknowledgements}

The authors would like to thank the Director, the technical staff and workers of the CRIA of Fouarat who contributed to the collection of semen data.

\section{References}

Amann RP, Almquist JO (1976) Bull management to maximize sperm output. In: Proc 6th Tech Conf Artif Insemination Reprod, National Association of Animal Breeders, Columbia, MO, USA, 1-10

Boujenane I (2002) [Cattle Breeds in Morocco]. Actes Editions, Rabat, Morocco [in French]

Brito LFC, Silva AEDF, Rodrigues LH, Vieira FV, Deragon LAG, Kastelic JP (2002) Effects of environmental factors, age and genotype on sperm production and semen quality in Bos indicus and Bos taurus Al bulls in Brazil. Anim Reprod Sci 70, 181-190

Everett RW, Bean B (1982) Environmental Influences on Semen Output. J Dairy Sci 65, 1303-1310

Fuerst-Waltl B, Schwarzenbacher H, Perner C, Sölkner J (2006) Effects of age and environmental factors on semen production and semen quality of Austrian Simmental bulls. Anim Reprod Sci 95, 27-37

Haugan T, Reksen O, Gröhn YT, Kommisrud E, Ropstad E, Sehested E (2005) Seasonal effects of semen collection and artificial insemination on dairy cow conception. Anim Reprod Sci 90, 57-71

Karoui S, Díaz C, Serrano M, Cue R, Celorrio I, Carabaño MJ (2011) Time trends, environmental factors and genetic basis of semen traits collected in Holstein bulls under commercial conditions. Anim Reprod Sci $124,28-38$

Mathevon M, Buhr MM, Dekkers JCM (1998) Environmental, Management, and Genetic Factors Affecting Semen Production in Holstein Bulls. J Dairy Sci 81, 3321-3330

SAS (2002) Release 9.2 Raleigh, SAS Institute Inc., Cary, NC, USA

Taylor JF, Bean B, Marshall CE, Sullivan JJ (1985) Genetic and Environmental Components of Semen Production Traits of Artificial Insemination Holstein Bulls. J Dairy Sci 68, 2703-2722

Teixeira HCA, Nascimento NV, McManus C, Egito AA, Mariante AS, Ramos AF (2011) Seasonal influence on semen traits and freezability from locally adapted Curraleiro bulls. Anim Reprod Sci 125, 56-61 